\title{
Assessment of enthesitis in patients with psoriatic arthritis using clinical examination and ultrasound
}

\author{
Salome Kristensen ${ }^{1}$ \\ Jeppe Hagstrup Christensen ${ }^{2}$ \\ Erik Berg Schmidt ${ }^{3}$ \\ Jens Lykkegaard Olesen ${ }^{4}$ \\ Martin Berg Johansen 5 \\ Kristian Bakke Arvesen ${ }^{6}$ \\ Annette Schlemmer ${ }^{1}$ \\ 1 Department of Rheumatology, Aalborg University \\ Hospital, Aalborg, Denmark \\ 2 Department of Nephrology, Aalborg University \\ Hospital, Aalborg, Denmark \\ 3 Department of Cardiology, Aalborg University \\ Hospital, Aalborg, Denmark \\ 4 Institute of Sports Medicine, Bispebjerg Hospital, \\ Copenhagen, Denmark \\ 5 Department of Cardiology and Unit of Clinical \\ Biostatistics and Bioinformatics, Aalborg University \\ Hospital, Aalborg, Denmark \\ 6 Department of Dermatology, Aarhus University \\ Hospital, Aarhus, Denmark
}

\section{Corresponding author:}

Salome Kristensen

Department of Rheumatology, Aalborg University

Hospital

Reberbansgade 15

9000 Aalborg, Denmark

E-mail:sakr@rn.dk

\section{Summary}

Background: Enthesitis is a major feature of psoriatic arthritis. However, clinical assessment of enthesitis is known to lack accuracy and have poor interobserver reliability.

Objective: To determine effect of training on clinical assessment of enthesitis and to compare ultrasonography with clinical examination for the detection of entheseal abnormalities.

Methods: 20 rheumatologists performed repeated assessment of enthesitis in patients with established psoriatic arthritis before and after a 2-hour training session in standardised enthesitis count according to Leeds Enthesitis Index (LEI) and Spondyloarthritis Research Consortium of Canada Enthesitis Index (SPARCC). Moreover, 20 pa- tients underwent clinical and ultrasonographic examination of entheses to evaluate consensusbased elementary lesions of enthesitis.

Results: Training significantly increased Intraclass Correlation Coefficient for LEI from 0.18 to 0.82 and for SPARCC from 0.38 to 0.67 . Ultrasound examination showed high associations between hypoechogenicity and increased thickness of the entheses and clinical examination. There was no correlation between erosions and enthesophytes found by ultrasound and clinical assessments.

Conclusion: Training in standardised enthesitis scoring systems significantly improved clinical assessments of enthesitis and should be performed before use in daily clinical practice. Ultrasound revealed more advanced stages of enthesitis, such as enthesophytes and erosions, which were not detected with clinical examination.

KEY WORDS: enthesitis, leeds enthesitis index, psoriatic arthritis, SPARCC, ultrasound.

\section{Introduction}

One of the key pathological features of psoriatic arthritis (PSA) is enthesitis. Enthesitis is defined as inflammation at the insertion of the tendons, ligaments, and capsules into bone. Recent registry and clinical trials have reported enthesitis in 30 to $50 \%$ of PsA patients ${ }^{1}$.

The OMERACT 7 International Consensus Conference on Outcome Measures in Rheumatology recognized the clinical importance of enthesitis, in addition to the assessment of peripheral joint disease, in $\mathrm{PsA}^{2}$. Enthesitis should be measured as an indicator of disease activity and treatment response in patients with PsA. So far, very few clinical trials in patients with PsA have determined the degree of enthesitis, and methods have typically not been validated for use in patients with $\mathrm{PsA}^{2}$.

Conventionally, enthesitis has been assessed by clinical examination and several enthesitis assessment tools exist (Tab. I). Latest Spondyloarthritis Research Consortium of Canada enthesitis index (SPARCC) and Leeds enthesitis index (LEI) have been developed. SPARCC was created as a measure for enthesitis in spondyloarthritis in general (not limited to PsA or Ankylosing Spondylitis) ${ }^{4}$ and has not been exten- 
Table I. Enthesial sites assessed in outcome measures for enthesitis.

\begin{tabular}{llll}
\hline & MASES & SPARCC & LEI (Leeds) \\
\hline First costochondral & $\mathrm{R}, \mathrm{L}$ & & \\
Seventh costochondral & $\mathrm{R}, \mathrm{L}$ & $\mathrm{R}, \mathrm{L}$ & $\mathrm{R}, \mathrm{L}$ \\
Supraspinatus insertion & & $\mathrm{R}, \mathrm{L}$ & $\mathrm{R}, \mathrm{L}$ \\
Lateral epicondyle humerus & & \\
Medial epicondyle humerus & $\mathrm{R}, \mathrm{L}$ & \\
Posterior superior iliac spine & $\mathrm{R}, \mathrm{L}$ & \\
Anterior superior iliac spine & $\mathrm{R}, \mathrm{L}$ & $\mathrm{R}, \mathrm{L}$ \\
Iliac crest & $\mathrm{X}$ & $\mathrm{R}, \mathrm{L}$ \\
Fifth lumbar spinous process & $\mathrm{R}, \mathrm{L}$ & $\mathrm{R}, \mathrm{L}$ \\
Achilles tendon & & $\mathrm{R}, \mathrm{L}$ \\
Greater trochanter & & $\mathrm{R}, \mathrm{L}$ \\
Medial condyle femur & & $\mathrm{R}, \mathrm{L}$ \\
Insertion plantar fascia & & $(\mathrm{R}, \mathrm{L})$ \\
Quadriceps insertion patella & & $\mathrm{R}$ \\
Inferior pole patella & & & \\
(Tibial turbercle) & & &
\end{tabular}

MASES: Maastricht Ankylosing Spondylitis Enthesis Score; SPARCC: Spondyloarthritis Research Consortium of Canada; LEI: Leeds Enthesitis Index; X: single site present, not bilateral; R: right; L: left.

sively validated in $\mathrm{PSA}^{5}$. LEI has been developed for measuring enthesitis in patients with $\mathrm{Ps}^{6}$. However, all the scoring systems are known to have limitations in terms of reliability, validity, and sensitivity ${ }^{3}$. Another limitation of clinical enthesitis count is the specificity of the finding of tenderness in these areas. Many of the entheseal points are relatively near to joints and accepted tender points for fibromyalgia, raising the possibility of misclassification.

Imaging modalities such as ultrasound (US) have been investigated to determine whether they improve entheseal disease assessment. Studies have shown that US indices for enthesitis are more sensitive than clinical examination 7,8 . The OMERACT US Specialist Interest Group has achieved agreement on US definition of enthesitis and its elementary components to ensure a higher degree of homogeneity and comparability of results between studies and in daily clinical work $^{9}$. However, the value of US findings at the enthesis is not investigated fully. Although Doppler sign at the enthesis is found more frequently in patients with PsA, as compared to healthy controls ${ }^{10}$, it can also be seen in patients with rheumatoid arthritis ${ }^{11}$. Bone changes such as enthesophytes and erosions may also be found as degenerative changes in weight-bearing entheses ${ }^{12}$. Furthermore, the application of US in daily practice in patients with PSA is limited by the time required to examine multiple sites of enthesitis.

Since clinical enthesitis is a hallmark feature of PsA, there is a need for improvement of clinical assessment of enthesitis and evaluation of the benefits of
US as an outcome measure in daily practice.

The aim of this study was to examine if training in standardised assessment of enthesitis according to LEI and SPARCC is able to improve interobserver variation in patients with established PsA. Furthermore, we aimed to compare US and clinical assessment of enthesitis to determine the added value of US in enthesitis assessment.

\section{Methods}

\section{Subjects}

Outpatients with PsA according to Classification Criteria for Psoriatic Arthritis (CASPAR criteria) were enrolled from the Departments of Rheumatology, Aalborg University Hospital, Denmark, during routine presentation to the clinic. The inclusion criteria were established PsA requiring systemic treatment with disease modifying anti-rheumatic drugs. Exclusion criteria were treatment with biological drugs or treatment with oral corticosteroids. All patients taking NSAIDs were asked to stop taking their medication a minimum of 24 hours before clinical and US assessments to avoid the immediate analgesic and anti-inflammatory response to NSAID and in order to compare results with previous studies ${ }^{12-15}$. All participants gave their informed consent, and the study was approved by the regional ethics committee, Northern Region Denmark. Furthermore the study meets the ethical standards of the journal ${ }^{16}$. 


\section{Clinical assessment with instruction session}

Four patients and one healthy control were assessed for enthesitis with the SPARCC index and LEI by 20 rheumatologists. All the rheumatologists obtained enthesitis scores in patients and the control subject before and after a formal group training session to validate the effect of training. In the training session a senior rheumatologist experienced in enthesitis scoring systems, demonstrated enthesitis assessment in a patient using the SPARCC index and LEI. Enthesitis was defined as tenderness at the site of an enthesis with a standard palpation approach, applying $\sim 4$ $\mathrm{kg} / \mathrm{cm}^{2}$ pressure (enough to blanch the tip of the examiner's fingernail). Tenderness on examination was recorded as either present (1) or absent (0) for each of the sites. The sites of examination are described in Table I. Higher scores reflect greater enthesitis burden. The healthy control was assessed to evaluate the potential risk of higher scores at the second assessment after the training session.

\section{US assessment compared to clinical assessment}

In a different setting, 20 patients (the 4 patients mentioned above and another 16 patients) with PsA were examined with US [Grey Scale (GS) and power Doppler (PD)] by a rheumatologist experienced in sonography (with over 8 years' experience in musculoskeletal US). Another trained rheumatologist performed the clinical examination on the same day as US scans. The patients underwent examination with enthesitis score using LEI and SPARCC, psoriasis severity was scored using the Psoriasis Area and Severity Index (PASI) and Disease Activity Score DAS66/68 (CRP) was calculated. The sonographer was blinded to the clinical examination findings of the patients. A HITACHI HA710 machine with an $18 \mathrm{MHz}$ linear probe $\mathrm{L} 75$ was used for examination of all patients. PD was assessed using a pulse repetition frequency of $750 \mathrm{~Hz}$ and medium wall filter, and gain was adjusted until background signal was removed. The sonographic assessments were made at each of the LEI and SPARCC enthesis sites except for the trochanter major region. US of trochanter major region acquires another probe and therefore data cannot be compared with findings at other sides. Each tendon was scanned in both the longitudinal and transverse planes. The following sites were evaluated bilaterally: supraspinatus insertion, lateral and medial epicondyle, medial condyle of the femur, superior and inferior pole of the patella, Achilles tendon insertion, and plantar aponeurosis insertion. The patients were positioned as in previous studies ${ }^{12,17}$. The supraspinatus tendon insertion was examined with the patient seated and the hands resting on the knee. The lateral and medial epicondyle insertions were evaluated with the patient seated, the hand resting on the knee with the elbow slightly flexed, and the wrist in slightly internal rotated. Knee enthesis examination was performed with the patient in the supine position and the knee flexed at $70^{\circ}$. The Achilles tendon and the plantar aponeurosis were examined with the patient lying prone and the feet hanging over the edge of the examination table at $90^{\circ}$ of flexion.

The US exploration evaluated the following lesions at each site:

1. Hypoechogenicity and increased thickness of the tendon insertion

2. Enthesophytes

3. Calcifications

4. Erosions

5. Power Doppler signal at enthesis (PD).

These 5 parameters were recently suggested and defined by the European League Against RheumatismOMERACT US Group ${ }^{18}$. Entheseal thickening was scored as present if there was a discrepancy in the thickness at the contralateral enthesis or if the normal entheseal contour appeared bulky. No attempt was made to measure the thickness of the enthesis due to difficulties in standardizing the measurement site. US score of enthesitis was calculated as follows: one point was scored for each abnormality at each site examined, giving a possible total score of 80 points on both sides.

Sonographic images were stored and rescored blindly by the investigator.

\section{Statistical analyses}

All statistical analyses were performed using Stata: Release 13 (Stata Corp LP, TX, US). The data on clinical examination before and after the instruction session are presented as the mean and range of the scores. The interobserver variability of the physicians' assessments of SPARCC index and LEI before and after the training session was assessed in an analysis of Intraclass Correlation Coefficients (ICC) in a twoway random effects model. Bootstrapping test ${ }^{19}$ was used to compare the ICC of the SPARCC index and LEI before and after instruction. Regarding the US examination, scatter plots and Spearman correlation coefficients were used to assess the relationships between each score system and US outcomes and health assessment (CRP and DAS66/68). Differences were considered significant with a $p$-value of $<0.05$ (two-tailed).

\section{Results}

\section{Patient charachteristics}

Twenty patients with PsA participated in the study. The patients had a mean \pm SD age of $49 \pm 9.9$ years, mean $\pm S D$ disease duration of $18.1 \pm 2.3$ years, mean \pm SD disease activity score (DAS) of $2.9 \pm 1.0$, mean \pm SD PASI of $3.1 \pm 3.8,74.8 \%$ were in therapy with methotrexate and $50.3 \%$ used NSAID on weekly basis. 
Table II. Mean score and Intraclass Correlation Coefficients for LEI and SPARCC Enthesitis Index before and after instruction.

\begin{tabular}{llll}
\hline & Before instruction & After instruction & Comparing ICCs before and after instruction \\
\hline Results & ICC $(95 \% \mathbf{C l})$ & ICC $(95 \% \mathbf{C I})$ & Bootstrap Z $(\boldsymbol{p}$-values $)$ \\
LEI & $0.18(0.03-0.79)$ & $0.82(0.51-0.99)$ & $2.85(0.004)$ \\
SPARCC & $0.38(0.12-0.90)$ & $0.67(0.35-0.97)$ & $2.17(0.03)$ \\
\hline
\end{tabular}

LEI: Leeds Enthesitis Index; SPARCC: Spondyloarthritis Research Consortium of Canada; ICC: Intraclass Correlation Coefficient; $\mathrm{Cl}$ : Confidence Interval.

\section{Enthesitis score and interobserver reliability}

Comparing ICCs for LEI and SPARCC index before and after training showed significantly higher ICC after training for both indices. ICC for LEI increased from 0.18 to 0.82 and for SPARCC from 0.38 to 0.67 (Tab. II).

An increase in both SPARCC and LEI enthesitis scores were found after training. LEI score increased by 1.95 points $(95 \%$ Confidence Interval [Cl] $1.47-$ 2.43) and SPARCC score increased by 1.78 points (95\% CI 1.16-2.39). The control person had no enthesitis before or after training. LEI and SPARCC index revealed only a moderate correlation with DAS66/68 ( $r=0.50$ for LEI and $r=0.34$ for SPARCC index), and there were no significant correlations between CRP levels and the two enthesitis scores.

\section{Correlation between clinical assessments and US findings}

US results showed a moderate correlation between total US score and LEI $(r=0.50)$ and SPARCC index $(r=0.47)$ (Figs. 1, 2). Looking at the different US parameters, there was high correlation between hypoechogenicity and tendon thickness and LEI $(r=0.81)$ and SPARCC score was also significantly correlated with hypoechogenicity and tendon thickness ( $r=$ $0.86)$. There were no correlations between PD and LEI and SPARCC.

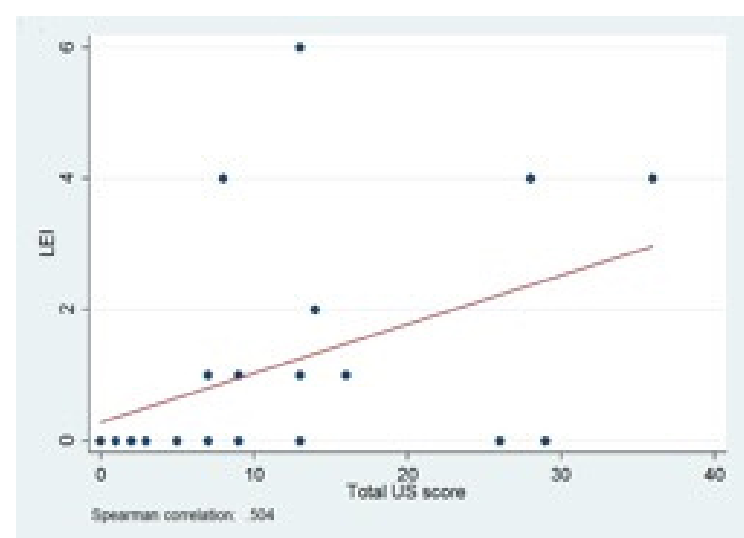

Figure 1. Correlation between LEI and total US score. LEI: Leeds Enthesitis Index; US: ultrasound.

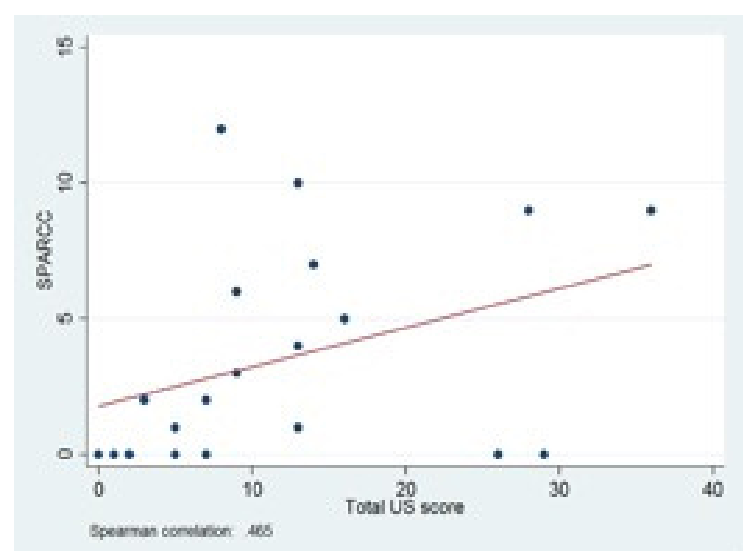

Figure 2. Correlation between SPARCC Enthesitis Index and total US score.

SPARCC: Spondyloarthritis Research Consortium of Canada; US: ultrasound.

More chronical findings such as enthesophytes and erosions were not correlated with clinical score with LEI and SPARCC. All correlation coefficients are given in Table III.

In addition, there was no correlation between the US score of enthesitis and CRP.

\section{Discussion}

Enthesitis occurs in approximately $48 \%$ of patients with PsA and at first visit, approximately $35 \%$ of the patients present with enthesitis ${ }^{20}$. Several clinical and US outcome measures for enthesitis in patients with PsA exists; however, their clinical use may be limited by time restraints and lack of training and consensus in the use of these tools ${ }^{18,21}$.

The present study showed a significant increase in ICC after training for both LEI and SPARCC scores. It is important to note that the ICCs obtained before the training session were very low. Furthermore, the increase in reliability after the training session suggests the need for training in standardised enthesitis counts before using such indices in clinical practice. Similarly, both LEI and SPARCC enthesitis scores increased significantly after training, reflecting an increased ability to detect enthesitis burden. 
Table III. Correlation between US findings and LEI and SPARCC Enthesitis Index.

\begin{tabular}{lll}
\hline US parameters & LEI & SPARCC \\
\hline Hypo-echogenicity and tendon thickness & $r=0.81^{\star *}$ & $r=0.86^{* *}$ \\
Calcification & $r=0.45^{*}$ & $r=0.22$ \\
Enthesophytes & $r=0.09$ & $r=0.05$ \\
Erosions & $r=0.05$ & $r=0.12$ \\
Power Doppler & $r=0.26$ & $r=-5.5 e-03$ \\
\hline
\end{tabular}

LEI: Leeds Enthesitis Index; SPARCC: Spondyloarthritis Research Consortium of Canada; r: Spearmans Correlation Coefficient; ${ }^{*}: p<0.05 ;{ }^{* *}: p<0.01$.

US examination in this study revealed a significant correlation between clinical enthesitis scores and US findings of hypoechogenicity and tendon thickness. However, examination did not detect bone changes found using US.

Comparing LEI to SPARCC, both scores were similar in aspect of ICC and detection of enthesitis burden before and after instruction. Additionally both scores correlated similarly with US findings.

The poor association between PD and enthesitis scores in our study might be explained by the fact that there are fewer vessels in inflamed enthesis compared with synovium making it harder to visual$i^{22,23}$. US examinations was assessed in positions previously described ${ }^{12}$ but not fully relaxed positions, and this may also contribute to reduction of the sensitivity of PD ${ }^{24-27}$. Previous studies by Gutierrez et al. ${ }^{28}$, Koenig et al. ${ }^{29}$ and Zappia et al. ${ }^{30}$ revealed changes in intratendinous PD related to joint position. The position of the joints for the evaluation of PD at the enthesis is an important limitation of this study. Examination positions for US should be studied to evaluate the optimal position for PD. Only few patients had PD at the enthesis; therefore, it was not possible to evaluate at which site the PD was better correlated to clinical examination. Furthermore, the small study population could have affected our results. The US examination was not performed in a control group, and some of the findings may be due to degenerative changes developed with age. However, the study group was relatively young.

In addition, a relatively small number of patients was clinically assessed for enthesitis before and after instruction and there is a need for further studies to validate specific enthesitis scores and to determine the value of formal instruction and training.

Interobserver variation in joint count scores has previously been described and training has been found to result in a reduction in the interobserver variability of joint counts ${ }^{31}$. This study indicates the need for training in enthesitis scores in patients with PsA in the daily clinical settings. Our training exercise was brief and informal, yet it was followed by a significant reduction in the interobserver variation.

Both enthesitis scores were only moderately associated with DAS66/68. As outpointed in previous stud- ies DAS66/68 will likely underestimate the burden of disease in PSA and it's multiple domains should be assessed 32,33 . We found no correlation between CRP levels and enthesitis scores. In a previous study of ankylosing spondylitis enthesitis was only correlated to high sensitive CRP and not routine CRP test ${ }^{34}$, indicating minimal influence of enthesitis on CRP. Overall CRP seems to be a more important marker of disease activity in rheumatoid arthritis ${ }^{35}$ than in $\mathrm{PsA}^{36}$.

US findings in enthesitis are often split into features of soft-tissue inflammation (hypoechogenicity, tendon thickness and PD) and features of tissue damage (bone changes) to reflect the reversible and irreversible pathological components of enthesitis. Similar to a previous study ${ }^{17}$, our US results suggests that enthesophytes and erosions are more chronic findings, not detectable with clinical examination. However, in a recent study of the OMERACT US Task Force it was not possible to reach an agreement on a definition of how to separate acute inflammation from chronic bone changes ${ }^{37}$. Furthermore, a previous study have shown that calcifications and enthesophytes found using US might be a common pathology in trauma and degenerative changes in the general population increasing with age ${ }^{38}$. Another issue to consider when using US for enthesitis is the correlation between US findings and disease activity and response to therapy. In a 6-month study of 197 patients with various spondylarthropaties (including PsA) the Authors demonstrated improvement in the morphologic abnormalities of the tendon as well as PD, but there were no changes in calcifications or erosions ${ }^{39}$. Further longitudinal studies in larger PsA populations are needed to decide how to interpret and use US findings.

In conclusion, this study shows that a two hour training session were able to significantly improve the reliability of the enthesitis assessment. Enthesitis score training in a standardized examination technique could potentially minimize interobserver variation. In addition, US may be more sensitive for the evaluation of bone changes, but further longitudinal studies are needed to determine if these findings correlate with disease activity and response to treatment. Since LEI is less time-consuming than SPARCC index, it might be more feasible to use in daily practice. 


\section{Acknowledgement}

The Authors acknowledge the patients participating in this study.

\section{Conflicts of interest}

The Authors declare no conflicts of interest concerning this article.

\section{Funding}

This article was not supported by research funding.

\section{References}

1. Gladman DD, Chandran V. Observational cohort studies: lessons learnt from the University of Toronto Psoriatic Arthritis Program. Rheumatology. 2011;50(1):25-31.

2. Gladman DD, Strand V, Mease PJ, Antoni C, Nash P, Kavanaugh A. OMERACT 7 psoriatic arthritis workshop: synopsis. Ann Rheum Dis. 2005;64 Suppl 2:115-116.

3. Mease PJ. Measures of psoriatic arthritis: Tender and Swollen Joint Assessment, Psoriasis Area and Severity Index (PASI), Nail Psoriasis Severity Index (NAPSI), Modified Nail Psoriasis Severity Index (mNAPSI), Mander/Newcastle Enthesitis Index (MEI), Leeds Enthesit. Arthritis Care Res. 2011;63 Suppl 1:S64-85.

4. Maksymowych WP, Mallon C, Morrow S, Shojania K, Olszynski WP, Wong RL, et al. Development and validation of the Spondyloarthritis Research Consortium of Canada (SPARCC) Enthesitis Index. Ann Rheum Dis. 2009;68(6):948-953.

5. Gladman DD, Inman RD, Cook RJ, et al. International spondyloarthritis interobserver reliability exercise - the INSPIRE study: II. Assessment of peripheral joints, enthesitis, and dactylitis. J Rheumatol. 2007;34(8):1740-1745.

6. Healy PJ, Helliwell PS. Measuring clinical enthesitis in psoriatic arthritis: assessment of existing measures and development of an instrument specific to psoriatic arthritis. Arthritis Rheum. 2008;59(5):686-691.

7. Balint P V, Kane D, Wilson H, McInnes IB, Sturrock RD. Ultrasonography of entheseal insertions in the lower limb in spondyloarthropathy. Ann Rheum Dis. 2002;61(10):905-910.

8. Gandjbakhch F, Terslev L, Joshua F, Wakefield RJ, Naredo E, D'Agostino MA. Ultrasound in the evaluation of enthesitis: status and perspectives. Arthritis Res Ther. 2011;13(6):R188.

9. Wakefield RJ, Balint P V, Szkudlarek M, et al. Musculoskeletal ultrasound including definitions for ultrasonographic pathology. J Rheumatol. 2005;32(12):2485-2487.

10. Aydin SZ, Ash ZR, Tinazzi I, et al. The link between enthesitis and arthritis in psoriatic arthritis: a switch to a vascular phenotype at insertions may play a role in arthritis development. Ann Rheum Dis. 2012;992-995.

11. lagnocco A, Spadaro A, Marchesoni A, et al. Power Doppler ultrasonographic evaluation of enthesitis in psoriatic arthritis. A multi-center study. Joint Bone Spine. 2012;79(3):324-325.

12. Freeston JE, Coates LC, Helliwell PS, et al. Is there subclinical enthesitis in early psoriatic arthritis? A clinical comparison with power doppler ultrasound. Arthritis Care Res. 2012;64(10): 1617-1621.

13. Soriano ER, McHugh NJ. Therapies for peripheral joint disease in psoriatic arthritis. A systematic review. J Rheumatol. 2006;33(7):1422-1430.
14. Mouterde G, Aegerter P, Correas JM, Breban M, D'Agostino MA. Value of contrast-enhanced ultrasonography for the detection and quantification of enthesitis vascularization in patients with spondyloarthritis. Arthritis Care Res. 2014;66(1): 131-138.

15. Davies NM. Clinical pharmacokinetics of ibuprofen. The first 30 years. Clin Pharmacokinet. 1998;34(2):101-154.

16. Padulo J, Oliva F, Frizziero A, Maffulli N. Muscles, Ligaments and Tendons Journal. Basic principles and recommendations in clinical and field science research: 2016 update. Muscles Ligaments Tendons J. 2016;6(1):1-5.

17. Balint P V, Kane D, Wilson H, McInnes IB, Sturrock RD. Ultrasonography of entheseal insertions in the lower limb in spondyloarthropathy. Ann Rheum Dis. 2002;61:905-910.

18. Terslev L, Naredo E, lagnocco A, Balint P V., Wakefield RJ, Aegerter $\mathrm{P}$, et al. Defining enthesitis in spondyloarthritis by ultrasound: Results of a delphi process and of a reliability reading exercise. Arthritis Care Res. 2014;66:741-748.

19. Carpenter J, Bithell J. Bootstrap confidence intervals: when, which, what? A practical guide for medical statisticians. Stat Med. 2000;19(9):1141-1164.

20. Gladman DD. Clinical Features and Diagnostic Considerations in Psoriatic Arthritis. Rheum Dis Clin North Am. 2015;41 (4):569-579.

21. Ferguson EG, Coates LC, Coates LC. Optimisation of rheumatology indices: dactylitis and enthesitis in psoriatic arthritis. Clin. Exp. Rheumatol. 2014;32(Suppl. 85):113-117.

22. Coates LC, Hodgson R, Conaghan PG, Freeston JE. MRI and ultrasonography for diagnosis and monitoring of psoriatic arthritis. Best Pract Res Clin Rheumatol. 2012;26(6):805-822.

23. Torp-Pedersen ST, Terslev L. Settings and artefacts relevant in colour/power Doppler ultrasound in rheumatology. Ann Rheum Dis. 2008;67(2):143-149.

24. Oliva F, Via A, Maffulli N. Physiopathology of intratendinous calcific deposition. BMC Med. 2012;10(1):95.

25. Hinsley H, Nicholls A, Daines M, Wallace G, Arden N, Carr A. Classification of rotator cuff tendinopathy using high definition ultrasound. Muscles Ligaments Tendons J. 2014;4(3):391397.

26. Weinreb JH, Sheth C, Apostolakos J, et al. Tendon structure, disease, and imaging. Muscles Ligaments Tendons $\mathrm{J}$. 2014;4(1):66-73.

27. Galletti S. Sonoelastography in the diagnosis of tendinopathies: an added value. Muscles Ligaments Tendons J. 2015;5 (4):325-330.

28. Gutierrez M, Filippucci E, Grassi W, Rosemfet M. Intratendinous Power Doppler Changes Related to Patient Position in Seronegative Spondyloarthritis. J Rheumatol. 2015;37(5): 1057-1059.

29. Koenig MJ, Torp-Pedersen ST, Christensen R, et al. Effect of knee position on ultrasound Doppler findings in patients with patellar tendon hyperaemia (Jumper's knee). Ultraschall der Medizin. 2007;28(5):479-483.

30. Zappia M, Cuomo G, Martino MT, Reginelli A, Brunese L. The effect of foot position on Power Doppler Ultrasound grading of Achilles enthesitis. Rheumatol Int. 2016;36(6):871-874 .

31. Stamp LK, Harrison A, Frampton C, Michael M. The Journal of Rheumatology Does a Joint Count Calibration Exercise Make a Difference? Implications for Clinical Trials and Training. J Rheumatol. 2012;39(4):877-878.

32. Helliwell PS. Assessment of disease activity in psoriatic arthritis. 2015;44-47.

33. Coates L. Outcome Measures in Psoriatic Arthritis. Rheum Dis Clin North Am. 2015;41(4):699-710.

34. Poddubnyy D a, Rudwaleit M, Listing J, Braun J, Sieper J. Comparison of a high sensitivity and standard $\mathrm{C}$ reactive protein measurement in patients with ankylosing spondylitis and non-radiographic axial spondyloarthritis. Ann Rheum Dis. 
2010;69(7):1338-1341.

35. Spiegel TM, King W, Weiner SR, Paulus HE. Measuring disease activity: comparison of joint tenderness, swelling, and ultrasonography in rheumatoid arthritis. Arthritis Rheum. 1987;30:1283-1288

36. Daunt AO, Cox NL, Robertson JC, Cawley MI. Indices of disease activity in psoriatic arthritis. J R Soc Med. 1987;80:556558.

37. Terslev L, Naredo E, lagnocco A, Pv B, Rj W, Aegerter P, et al Defining enthesitis in spondyloarthritis by ultrasound: Results of a Delphi process and of a reliability reading exercise. Arthritis Care Res. 2014;66(5):741-748.

38. Benjamin M, McGonagle D. Histopathologic changes at "synovio-entheseal complexes" suggesting a novel mechanism for synovitis in osteoarthritis and spondylarthritis. Arthritis Rheum. 2007;56(11):3601-3609.

39. Naredo E, Batlle-Gualda E, Garcia-Vivar ML, et al. Power doppler ultrasonography assessment of entheses in spondyloarthropathies: Response to therapy of entheseal abnormalities. J Rheumatol. 2010;37(10):2110-2117. 